\title{
Vulvar Lymphangiosarcoma in a Bitch
}

\author{
Isabella de Almeida Fabris', Marconi Rodrigues de Farias'², Juliana Werner ${ }^{3}$, \\ Vinicius Gonzalez Peres Albernaz', Taíse Fuchs" ${ }^{4}$ Liv Cristina Miara² \& Juliany Gomes Quitzan'
}

\begin{abstract}
Background: Lymphangiosarcoma (LSA) is a rare, highly malignant and infiltrative neoplasm of the lymphatic endothelium of dogs and cats. It is mostly reported in medium to large breed dogs, over 5-year-old, with no sexual predisposition. Affected animals present fluctuating and diffuse swelling, covering both dermis and subcutaneous tissue, spreading through lymphatic and haematic vessels. Histologically, LSA is characterized by connected channels devoid of conspicuous haematic elements. Treatment depends on location of the neoplasm, staging, and possibility of curative surgical excision. Chemotherapy and radiotherapy can increase survival time. In this report, we describe a rare case of vulvar LSA in a dog. This is the first Brazilian report of LSA in dogs so far.

Case: A 3-year-old, female, mixed breed dog was presented for evaluation of vesicle-bullous lesions in the vulvar and perivulvar region with progressive growth along 6 month. Histopathology revealed neoplastic proliferation in the superficial dermis, advancing through the profound dermis. The histological lesion pattern was consistent with angiosarcoma, which united along with macroscopic pattern of the tumor, and the presence of multiple anastomosed vascular structures without erythrocytes within it at microscopy, was compatible with LSA. No evidence of metastasis or lymphadenopathy was found on survey radiography and ultrasound. We performed a surgical excision, and remaining wound was reconstructed with an advancement skin flap. Despite wide surgical resection, neoplastic cells could be found in surgical borders, as well as a metastatic inguinal lymph node. Postoperative chemotherapy based on doxorubicin as a single agent was administrated. Disease free interval (DFI) was one month after surgery, when small bullous lesions were observed near the surgical site, and histopathological exam confirmed LSA. Three months after the surgical procedure, the patient presented with worsening of the lesions, anorexia, and apathy. The owner opted for euthanasia. Total survival time was five months.

Discussion: The LSA occurs often in dogs above five years old, unlike this case, in which the patient was 3 years old. Several anatomic locations were cited in other reports. In this case, the lesion was in both the vulvar and perivulvar regions, which is an uncommon location of LSA, described as secondarily affected region in one report only. Despite absence of metastasis in this case, lungs, kidneys, bone marrow, and spleen can be affected. Histopathologic exam is the golden standard for a definitive diagnose of LSA. Diagnosing LSA may be challenging due to its resemblance to hemangiosarcoma, and confirmation is only possible if histopathology detects an absence of red blood cells in the lymphatic channels. Prognosis is poor due to LSA aggressive and infiltrative features. Doxorubicin demonstrated good clinical response in other dogs. Chemotherapy protocols, whether alone or in combination with cyclophosphamide, have been proving to be promising in dogs with soft tissue sarcomas, such as LSA. Surgical approach is the most appropriate and it may be associated with radiotherapy or chemotherapy, especially if complete resection of the tumor is not possible. In this report, even though it was a young animal, the location and extension of the lesion did not contribute to the good prognosis, even after adding adjuvant chemotherapy to the treatment.
\end{abstract}

Keywords: oncology, lymphangiosarcoma, dog, chemotherapy, doxorubicin. 


\section{INTRODUCTION}

The lymphangiosarcoma (LSA) is a rare, highly malignant and infiltrative neoplasm that originates from the cells of the lymphatic endothelium of dogs and cats [25]. It is mostly reported in medium to large breed dogs, over 5 years old, with no sexual predisposition [17,23].

Macroscopically, they are usually edematous and soft, and quickly infiltrate the underlying tissue, spreading through lymphatic and haematic vessels $[4,17]$. Affected animals present a fluctuating, diffuse, and sometimes painful swelling, covering both dermis and subcutaneous tissue [4].

In humans, its occurrence is usually related to the presence of chronic lymphedema [4]. It is suggested that the persistent presence of interstitial fluid rich in proteins or the chronic physical pressure in the lymphatic endothelium may be a stimulator for neoplastic transformation [6].

The golden standard for diagnose is histopathology. Histologically, LSA is characterized by connected channels devoid of conspicuous haematic elements, and coated by large and atypical endothelial cells through edematous collagen trabeculae [4]. The treatment depends on location of the neoplasm, clinical staging, and possibility of surgery with complete surgical excision [25]. Chemotherapy can increase survival time, but few articles describe different protocols and follow-ups in dogs $[3,8,11,12,18]$.

This report describes a case of a young female dog affected by LSA, unusually located in both vulvar and perivulvar region, submitted to surgical resection and adjuvant chemotherapy with doxorubicin. This is the first Brazilian case report of LSA in dogs so far.

\section{CASE}

A 3-year-old, unneutered, female mixed-breed dog, weighting $12 \mathrm{~kg}$, was presented for evaluation of vesicle-bullous lesions with progressive growth along 6 months. During physical exam, a painful, fluctuating bullous lesion characterized by an erythematous base, its sero-hemorrhagic content, and poorly defined dermographic margins was detected in the perivulvar region, extending to the vulvar, inguinal and medial thigh regions (Figure 1).

Initially, a fine needle aspiration for cytological examination revealed a hypercelular sample, contaminated with blood and characterized by the predomi- nance of lymphocytes, vacuolized macrophages, rare neutrophils, and no bacteria, characterizing inflammation. Fusiform cells with abundant cytoplasm, at times binucleated with sparse chromatin, evident nucleoli and presence of mitotic figures were visualized. Complete blood count and biochemical analysis were within normal limits for the specie. Thoracic radiography and abdominal ultrasound were performed and no evidence of signs compatible with metastasis or lymphadenopathy was found.

Subsequently, patient was submitted to incisional biopsy for histopathological exam. The microscopic aspect showed neoplastic proliferation in the superficial dermis, advancing through the profound dermis and a portion of panniculus adipose with poorly defined borders. The neoplastic cells were small, poorly differentiated, globular, and proliferated forming multiple anastomosed vascular structures, which were optically empty. There was stromal edema accompanied by mild infiltration of lymphocytes and plasma cells. Anisocariose, anisocytosis, and nuclear atypia were noted.

The mitotic index was $<1$ m.f./40x. The histological lesion pattern was consistent with angiosarcoma, which united along with macroscopic pattern of the tumor and the lack of erythrocytes within the vascular space at microscopy, was compatible with LSA (Figure 2).

A wide surgical excision was performed after histopathological results, and an advancement skin flap from the lateral face of the left hind limb was necessary to cover the surgical defect (Figure 3).

Despite wide surgical resection and adequate healing, a histopathological exam of the excised tissue revealed compromised margins and a metastatic inguinal lymph node. We decided to associate chemotherapy with doxorubicin $\left(30 \mathrm{mg} / \mathrm{m}^{2}\right)$ every three weeks. One month after surgical resection, small bullous lesions were observed near the surgical site, and histopathology confirmed the recurrence of the neoplasia. However, due to persistent hematological changes, chemotherapy was discontinued after the third cycle. Two months after the last session with doxorubicin, the patient returned with worsening of the clinical condition, presenting ulcerated lesions with serosanguineous fluid in the perivulvar and inguinal regions, anorexia, and apathy. The owner opted for euthanasia. Total survival time was five months. 


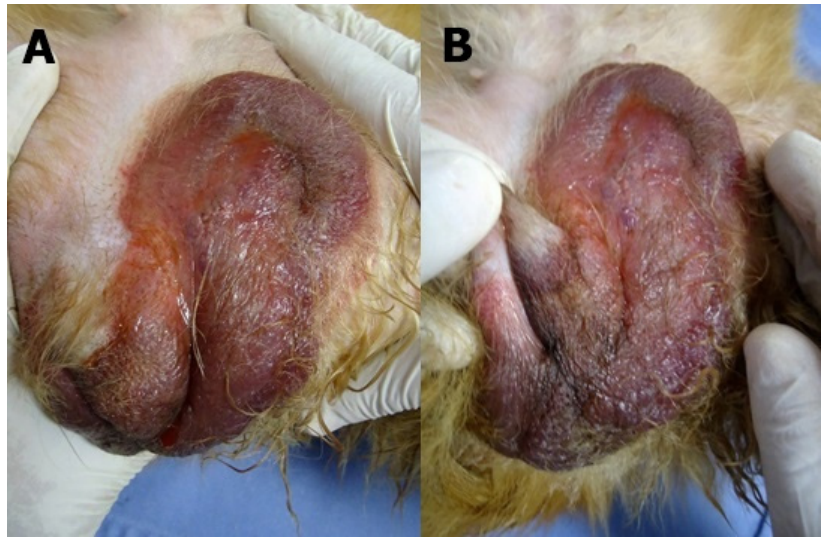

Figure 1. A and B- Images from physical exam showing bullous, erythematous and fluctuating aspect of the lesion.

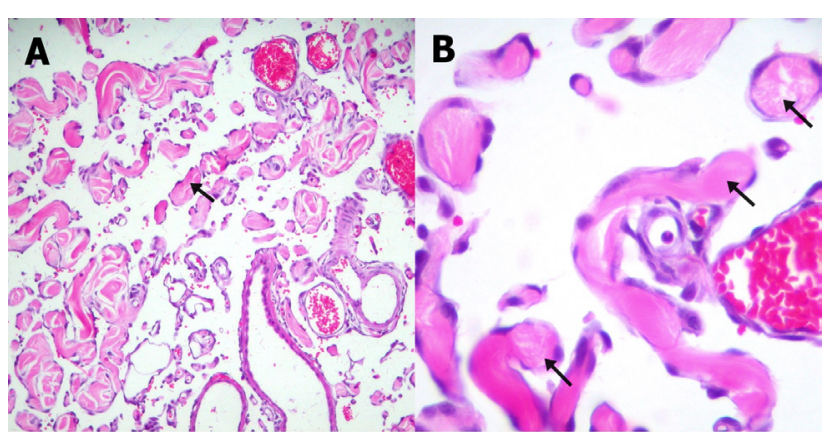

Figure 2. Photomicrograph of the lymphangiossarcoma describe in this report. A- Notice the disorganized proliferating mesenquimal cells forming multiple empty anastomosed vascular spaces (arrow) [H\&E, 50x]. B- Higher magnification of the left picture [H\&E, 400x].

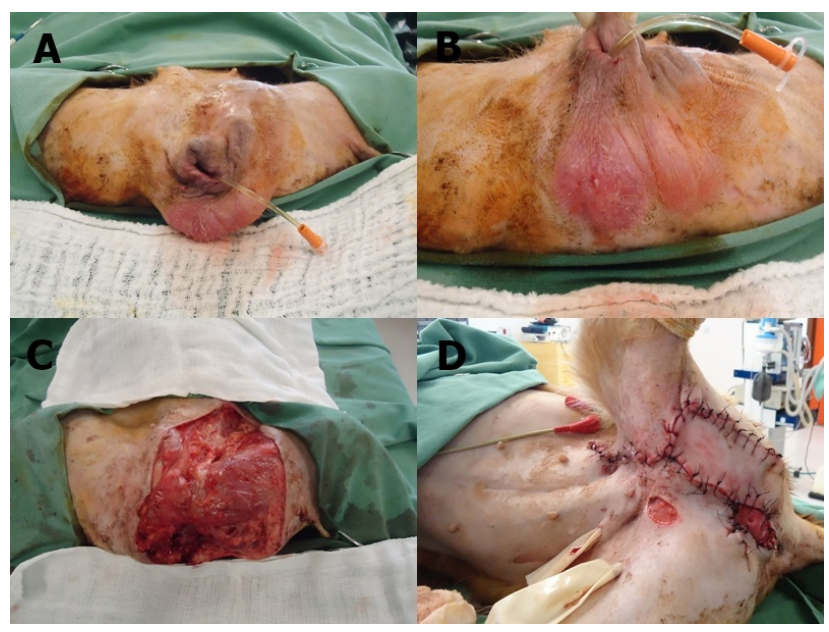

Figure 3. A and B- Pre-operative images evidencing the neoplasm as a fluctuating lesion with poorly defined margins; $\mathrm{C}$ - Trans-operative of wide tumor excision; D- Post-operative aspect of the site, after rotational skin flap.

\section{DISCUSSION}

LSA reports in dogs were published in England [12], Australia [9], United States [3,5,6,10,11,13,15,16,18,21], Argentina [4], Canada $[1,20,22]$, South Africa [24], Japan [8,14,19]. This is the first report of this neoplasm in Brazil so far.
It has been reported 33 cases of LSA until this moment [1,3-6,8-16,18-22,24]. There is no sexual predisposition for this kind of tumor. However, a retrospective study [23] revealed that most part of reported cases were males ( 9 males $-56.25 \%$ ). Nonetheless, in recent literature, females were more affected, in accordance with the present report (18 females - 55\%) $[3,4,9,12,14,16,19,21,24]$.

The predominant age group for this type of neoplasm is above five years old [3,8,10,12-16,19-21], unlike this case, in which the patient was three years old. There is no racial predisposition for LSA, but it has been noticed that it occurs more in medium to large breeds [1,3-6,8-10,12,13,16,20,21,24].

Several anatomic locations were cited, such as head, cervical region, and thoracic inlet $[3,5,6,8,14]$, inguinal region, prepuce and extremities $[3,10,11,16,18,22]$, axillar region $[1,3,4,9,16]$, thoracic subcutaneous tissue $[3,13,19]$, thoracic cavity and mediastinum [15,20,21], and caudal abdomen [12,24]. In this report, the lesion was in both the vulvar and perivulvar regions, similar to one report only, where vulvar area was secondarily affected [16]. The inguinal lymph node was affected and there were no signs of metastasis in other organs. However, lungs, kidneys, bone marrow, and spleen can be affected [4].

Image exams, such as radiography, abdominal ultrasound or computerized tomography (CT) and magnetic resonance imaging (MRI), can be done to search for metastatic lesions. Both CT and MRI can also assist surgical planning, as they determine wound limits [25].

Routine laboratory tests are performed, such as complete blood cells count, serum biochemistry [25], and urinalysis [3]. Results of blood samples are usually within normal reference values $[1,3,4,12,22]$, as in this case. Nonrtheless, authors report lymphopenia [8,20,21], neutrophilia [10,11], nonregenerative anemia [3], regenerative anemia [3,5], neutropenia [3], azotemia [3,10], hypokalemia [5], hyperkalemia [10], hypoproteinemia [5,10], hypoalbuminemia [5], and hyponatremia and hypercloremia [10] in animals with LSA.

Although fine needle aspiration has limited utility in the diagnose of LSA [25], this procedure not only helped excluding differential diagnoses such as abscesses and cysts, but also showed presence of cellular atypia and mitosis characteristic of neoplasm process.

Histopathologic exam is the golden standard for a definitive diagnose of LSA [12]. Excisional bi- 
opsy is not recommended because it is rarely curative, and the subsequent surgery to excise complete histological margins is generally more aggressive than the surgery followed by punch or incisional biopsy [25].

Diagnosing LSA may be challenging due to its resemblance to hemangiosarcoma, and confirmation relies on lack of erythrocytes within the vascular spaces [12], as well as in stromal edema with lymphoplasmacytic infiltration [7]. Immunohistochemistry may also help in differentiation of angiosarcoma of vascular origin from angiosarcoma of lymphatic origin. In humans, it is based on expression of CD44 and podocalyxin by the former versus lymphatic vessel endothelial receptor-1 (LYVE-1), podoplanin, and vascular endothelial growth factor receptor-3 (VEGFR-3) by the latter [7]. One study of 12 cases of lymphangiosarcoma in dogs confirmed the application of a lymphatic endothelial cell-specific marker, PROX-1, to differentiate between lymphangiosarcoma and hemangiossarcoma [3].

In this report, diagnose was achieved by means of histopathological exam, that showed similar findings described in literature about this neoplasm. Mitosis are not common in this tumor [4,9], but this patient presented intense neoplastic proliferation with no apparent demarcations, and with few differenced and globous cells, though small and with low mitotic rate. Empty vascular channels, typical of this neoplasm, were visualized on histopathological exam. Due to its aggressive and infiltrative characteristic, prognosis of animals presented with LSA is poor [25]. In this case, wide surgical excision was not enough to guarantee free margins. Additionally, the involvement of the inguinal lymph node associated with the presence of mitosis confirmed the invasive character of this neoplasm.

Adjuvant chemotherapy was added to treatment in order to increase the patient's survival time. There are eight cases described in literature which mention the use of chemotherapy in addition to surgical excision of LSA. The most used agent was doxorubicin $[3,8,11,12]$, followed by carboplatin [3], lomustin [3], mitoxantrone [20], metronomic with clorambucil [3, 12] and ciclophosphamide [3]. Radiotherapy was also used in one report [13], and it resulted in a four months of disease free interval (DFI), as shown in Table 1.

The chemotherapy agent chosen was doxorubicin, as it had demonstrated to have good clinical response

Table1. Chemotherapy protocols for LSA described in literature.

\begin{tabular}{|c|c|c|c|c|c|}
\hline $\begin{array}{l}\text { Reference } \\
\text { /year }\end{array}$ & Surgery & Agents/therapy cited & Number of sessions & $\begin{array}{l}\text { Disease free } \\
\text { interval }\end{array}$ & $\begin{array}{l}\text { Follow-up time } \\
\text { reported }\end{array}$ \\
\hline 17 (1996) & Yes & Derivate of doxorubicin & Six & Five months & Six months \\
\hline 7 (2004) & Yes & Doxorubicin & Four & Nine months & - \\
\hline 10 (2007) & Yes & $\begin{array}{l}\text { Radiotherapy associated } \\
\text { with doxorubicin }\end{array}$ & Four & Four months & Fifteen months \\
\hline $21(2012)$ & Yes & Mitoxantrone & Six & Ten months & Ten months \\
\hline $11(2013)$ & Yes & $\begin{array}{l}\text { (A) Doxorubicin } \\
\text { (B) Metronomic with } \\
\text { Clorambucil } \\
\text { (C) Metronomic with } \\
\text { Clorambucil + Palladia }\end{array}$ & $\begin{array}{l}\text { (A) Five } \\
\text { (B) Two months, } \\
\text { daily } \\
\text { (C) Two weeks } \\
\text { (interleaved days) }\end{array}$ & $\begin{array}{l}\text { (A) Six months } \\
\text { (B) Zero } \\
\text { (C) Regression } \\
\text { of tumor }\end{array}$ & $\begin{array}{l}\text { Fourteen } \\
\text { months }\end{array}$ \\
\hline $3(2014)$ & $\begin{array}{l}\text { 1) No } \\
\text { 2) Yes } \\
\text { 3) Yes }\end{array}$ & $\begin{array}{c}\text { Case 1: Doxorubicin } \\
\text { + ciclophosphamid } \\
\text { metronomic } \\
\text { Case 2: Carboplatine } \\
\text { Case 3: (A) Radiation } \\
\text { + doxorubicin and } \\
\text { lipossomal doxorubicin } \\
\text { (B) Lomustine (rescue } \\
\text { protocol) }\end{array}$ & $\begin{array}{l}\text { 1) Five } \\
\text { 2) Four } \\
\text { 3) (A) Four } \\
\text { 3) (B) One }\end{array}$ & $\begin{array}{l}\text { 1) } 182 \text { days } \\
\text { 2) } 75 \text { days } \\
\text { 3) (A) } 240 \text { days } \\
\text { 3) (B) Zero }\end{array}$ & - \\
\hline
\end{tabular}


in other dogs $[3,8,11,12,18]$. Chemotherapy protocols including doxorubicin, whether alone or in combination with cyclophosphamide, have been proving to be promising in dogs with soft tissue sarcomas, such as LSA [25], as it is demonstrated in a clinical study [3].

This patient had DFI of one month, during the chemotherapy treatment, and survival time was five months. The DFI was considered short when compared to other reports $[3,8,11,12,18]$ probably because of histopathologic features, lymph node involvement, and absence of free surgical margins. Fewer chemotherapy sessions were performed compared to other reports $[3,8,11,12,18]$, suggesting that longer protocols could improve remission time, between six and nine months. Other authors proposed the use of a similar protocol, after surgical resection of the mass, using doxorubicin as a single chemotherapic agent for four sessions [8]. Although free surgical margins were not achieved, the remission time was 9 months, but no metastatic site was found [8], unlike the results in this report.
In other protocols [3, 11], association of two or more chemotherapy agents were used to allow a superior time of remission of the disease/survival time in patients, especially if treatment is associated with radiotherapy.

Radiotherapy can be used as an adjuvant to surgery, whether with excision of complete margins or not, and it can be done in pre-op, post-op or as a single therapy, to treat and control pain [25]. However, this mode of therapy is not widely available yet.

Surgical approach is most appropriate and it may be associated with radiotherapy or chemotherapy, especially if full resection of the tumor is not possible [25]. In this report, although it was a young animal, the location and extension of the lesion, associated with malignancy of the neoplasm, did not contribute to a good prognosis, even after adding adjuvant chemotherapy to the treatment.

Declaration of interest. The authors report no conflicts of interest. The authors alone are responsible for the contents and writing of the paper.

\section{REFERENCES}

1 Barnes J.C., Taylor S.M., Clark E.G., Haines D.M. \& Broughton S.J. 1997. Disseminated lymphangiosarcoma in a dog. Canadian Veterinary Journal. 38(1): 42-44.

2 Chung K.C., Kim H.J.E. \& Jeffers L.L.C. 2000. Lymphangiosarcoma (Stewart-Treves syndrome) in postmastectomy patients. Journal of Hand Surgery (American Volume). 25(6): 1163-1168.

3 Curran K.M., Halsey C.H.C. \& Worley D.R. 2014. Lymphangiosarcoma in 12 dogs: a case series (1998-2013).Veterinary and Comparative Oncology. 14(2): 181-190.

4 Diessler M.E., Castellano M.C., Massone A.R., Portiansky E.L., Allende M.G., Idiart J.R. \& Gimeno EJ. 2003. Cutaneous lymphangiosarcoma in a young dog: Clinical, anatomopathological and lectinhistochemical description. Journal of veterinary medicine. A, Physiology, pathology, clinical medicine. 50(9): 452-456.

5 Fossum T.W., Miller M.W. \& Mackie J.T. 1998. Lymphangiosarcoma in a dog presenting with massive head and neck swelling. Journal of American Animal Hospital Association. 34(4): 301-304.

6 Franklin R.T., Robertson J.R. \& Thornburg L.P. 1984. Lymphangiosarcoma in a dog. Journal of the American Veterinary Medical Association. 184(4): 474-475.

7 Gross T.L., Ihrke P.J., Walder E.J. \& Affolter V.K. 2005. Interface Diseases of the dermal-epidermal junction. In: Gross T.L., Ihrke P.J., Walder E.J. \& Affolter V.K. (Eds). Skin diseases of the dog and cat: clinical and histopathologic diagnosis. 2nd edn. Oxford: Blackwell Publishing, pp.753-757.

8 Itoh T., Mikawa K., Mikawa M., Nibe K. \& Uchida K. 2004. Lymphangiosarcoma in a dog treated with surgery and chemotherapy. Journal of Veterinary Medical Science. 66(2): 197-199.

9 Kelly W.R., Wilkinson G.T. \& Allen P.W. 1981. Canine angiosarcoma (lymphangiosarcoma).Veterinary Pathology. 18(2): 224-227.

10 Lamb W.A. \& Muir P. 1994. Lymphangiosarcoma associated with hyponatraemia and hyperkalaemia in a dog. Journal of Small Animal Practice. 35(7): 374-376.

11 Looper J.S. 2007. Fat necrosis simulating recurrent neoplasia following external beam radiotherapy in a dog. Veterinary Radiology \& Ultrasound. 48(1): 86-88.

12 Marcinowska A., Warland J., Brearley M. \& Dobson J. 2013. A novel approach to treatment of lymphangiosarcoma in a boxer dog. Journal of Small Animal Practice. 54(6): 334-337. 
13 Messonnier S. 1990. Letter to the editor: lymphangiosarcoma. Journal of American Animal Hospital Association. 26(3): 242.

14 Mineshige T., Sugahara G., Ohmuro T., Kamiie J. \& Shirota K. 2015. Lymphangiosarcoma with bone formation of the auricle in a dog. Journal of Veterinary Medical Science. 77(6): 739-742.

15 Myers N.C., Engler S.J. \& Jakowski R.M. 1996. Chylothorax and chylous ascites in a dog with mediastinal lymphangiosarcoma. Journal of American Animal Hospital Association. 32(3): 263-269.

16 Rudd R.G., Veatch J.K., Whitehair J.G., Withrow S.J. \& Cook J.E. 1989. Lymphangiosarcoma in dogs. Journal of American Animal Hospital Association. 25: 695-698.

17 Ryan S., Wouters E.G.H., Nimwegen S. \& Kirpenstejin J. 2012. Skin and subcutaneous tumors. In: Kudnig S.T. \& Séguin B. (Eds). Veterinary Surgical Oncology. New York: John Wiley \& Sons, pp.55-85.

18 Sagartz J.E., Lairmore D.H., Sheafor S.E. \& Couto C.G. 1996. Lymphangiosarcoma in a Young Dog. Veterinary Pathology. 33(3): 353-356.

19Shiga A., Shirota K., Une Y. \& Nomura Y. 1994. Lymphangiosarcoma in a dog. Journal of Veterinary Medical Science. 56(6): 1199-1202.

20 Sicotte V., Benamou J., Fifle L.C., Planté J., Hugues I.L., Joly H. \& Stern A.W. 2012. Use of surgery and mitoxantrone chemotherapy in a dog with disseminate lymphangiosarcoma. Journal of the American Veterinary Medical Association. 241(12): 1639-1644.

21 Waldrop J.E., Pike F.S., Dulisch M.L., Ortega T.M. \& Gliatto J.M. 2001. Chylothorax in a dog with pulmonar lymphangiosarcoma. Journal of American Animal Hospital Association. 37(1): 81-85.

22 Webb J.A., Boston S.W., Armstrong J. \& Moens N.M.M. 2004. Lymphangiosarcoma Associated with Primary Lymphedema in a Bouvier des Flandres. Journal of Veterinary Internal Medicine. 18(1): 122-124.

23 Williams J.H. 2005. Lymphangiosarcoma of dogs: a review. Journal of the South African Veterinary Association. 76(3): $127-131$.

24 Williams J.H., Birrell J. \& Van Wilpec E. 2005. Lymphangiosarcoma in a 3.5-year-old Bullmastiff bitch with vaginal prolapse, primary lymph node fibrosis and other congenital defects. Journal of the South African Veterinary Association. 76(3): 165-171.

25 Withrow S.J., Vail D.M. \& Page R.L. 2013. Small Animal Clinical Oncology. 5th edn. St. Louis: Elsevier Saunders, pp.370-383. 